\title{
Laser Repetition Rate in Time-domain Thermoreflectance Techniques
}

\author{
L.S. Larkin $*, a$, J.L. Smoyer $*, b$, P.M. Norris $* *, a$ \\ ${ }^{a}$ Department of Mechanical and Aerospace Engineering, 122 Engineer's Way, \\ Charlottesville, VA, 29903 \\ ${ }^{b}$ Department of Physical Sciences, Kutztown University, Kutztown, PA 19530
}

${ }^{*}$ Corresponding author

${ }^{* *}$ Principal corresponding author

Email addresses: 1sl9hd@virginia.edu (L.S. Larkin $\left.{ }^{*, a}\right)$, jsmoyer@kutztown.edu (J.L. Smoyer *,b)

Preprint submitted to Elsevier

February 8, 2017

(C) 2016. This manuscript version is made available under the Elsevier user license http://www.elsevier.com/open-access/userlicense/1.0/ 


\title{
Laser Repetition Rate in Time-domain Thermoreflectance Techniques
}

\author{
L.S. Larkin $*, a$, J.L. Smoyer $*, b$, P.M. Norris $* *, a$ \\ ${ }^{c}$ Department of Mechanical and Aerospace Engineering, 122 Engineer's Way, \\ Charlottesville, VA, 29903 \\ ${ }^{d}$ Department of Physical Sciences, Kutztown University, Kutztown, PA 19530
}

\begin{abstract}
Pump-probe thermoreflectance techniques have been utilized extensively to measure the thermal properties; such as, thermal conductivity and interfacial conductances of thin film systems. While there have been many implementations of transient measurements, the most commonly operated systems utilize a near-IR femtosecond pulsed system as the fundamental laser source. The repetition rate of these femtosecond pulsed systems can vary from $\mathrm{kHz}$ to MHz. Thus, we perform an in-depth comparison of the implementation and analysis of low-repetition rate and high-repetition rate time-domain thermoreflectance (TDTR), demonstrating that despite varying the repetitionrate, the measured thermal properties are consistent. To illustrate the universality of these systems, we selected a range of materials to study on both TDTR set-ups, including the thermal conductivity of a $\mathrm{SiC}$ film, a $\mathrm{SiO}_{2}$ film, and the interfacial conductances of $\mathrm{Al} / \mathrm{SiO}_{2}$ and $\mathrm{Al} / \mathrm{SiC}$ systems.
\end{abstract}

Keywords: Time-domain Thermoreflectance, Thin film thermal properties

\section{Introduction}

Thermoreflective spectroscopy first appeared in the literature in the 1970s, where modulated continuous wavelength (CW) lasers were used to measure the photoacoustic spectra of solids and the thickness of opaque films $[1,2]$.

\footnotetext{
*Corresponding author

** Principal corresponding author

Email addresses: 1sl9hd@virginia.edu (L.S. Larkin $\left.{ }^{*}, a\right)$, jsmoyer@kutztown.edu (J.L. Smoyer *,b)
} 
Over the next decade, these non-contact techniques began to incorporate pico- and femtosecond pulsed lasers to investigate a range of phenomena, such as non-equilibrium dynamics [3, 4], optical constants [5], coherent acoustic phonons $[6,7]$, and heat diffusion in thin films $[8,9,5]$. Today, transient thermoreflective (TTR), or as it has come to be called, time-domain thermoreflectance (TDTR), is one of the most powerful and versatile techniques to extract thermal properties of multi-layered thin film systems $[10,11,12]$.

Most current TDTR systems use a high-power, high-repetition rate, Ti:sapphire oscillator as the primary pulsed laser source $[10,12,11]$. These systems further modulate the pump beam on the order of $100 \mathrm{~s}$ of $\mathrm{kHz}$ to several $\mathrm{MHz}$, which permits several successive heating events, with insufficient cooling time between events to return the system to equilibrium, during the "ON" portion of the modulation window. In this work, this type of system will be referred to as a high-repetition rate, or "high-rep", TDTR system. In order to better study non-equilibrium thermal phenomena, such as electron-phonon coupling, the basic high-repetition rate laser oscillator is often coupled with a regenerative amplifier to increase the pulse energy at the expense of the repetition rate $[13,14,15]$. These low-repetition rate systems typically operate at frequencies on the order of several 10s to several $100 \mathrm{kHz}$. In this scheme, only a single heating event occurs within the modulation window and the system is allowed to return to thermal equilibrium before the arrival of the next pump pulse. In the following work this one-shot-on/one-shot-off system is classified as a low-repetition rate, or "low-rep" TDTR system. In addition to non-equilibrium dynamics, recent work has shown low repetition systems are ideal for probing semiconductor surfaces directly [16].

Several prior studies have shown that the beam diameter and modulation frequency can impact the measured thermophysical properties in TDTR experiments, and care must be taken to ensure an accurate analysis is done [17, $18,19,20]$. Despite this collection of work, there has never been a work which has analyzed the overall repetition rate of the fundamental laser system. With an increasing number of facilities using TDTR, it is critical to establish the universality of the technique regardless of the repetition rate of the fundamental laser, a defining characteristic of a pulsed laser system. Therefore, we used two laser systems, varying only in repetition rate, one of high repetition rate $(80 \mathrm{MHz})$ and one of low repetition rate $(250 \mathrm{kHZ})$, to measure the thermal conductivity, $\kappa$, two materials (an oxide layer and a conductive substrate), and the thermal boundary conductance, $h_{B D}$, at two interfaces (a conductive metal/semiconductor, and a metal/oxide/semiconductor system). 


\section{Time-domain ThermoReflectance}

A basic schematic of a TDTR system, regardless of laser repetition rate, follows the layout in Fig. 1. A pulsed laser source is split into the high energy pump beam and the lower-energy probe beam. The pump beam is further modulated, through an electro-optical modulator (EOM) or equivalent equipment, to a frequency distinct from the fundamental laser frequency, and then directed onto the sample to induce a small heating event on the order of $1 \mathrm{~K}$. The probe is delayed temporally with respect to the pump via a mechanical delay stage, and then directed onto the sample.

The reflected probe beam from the sample surface is then collected via a photodetector and the resultant signal processed by a lock-in amplifier detection scheme. The reflectivity of a material is, in general, correlated to the temperature of the material through the thermoreflectance coefficient. Thus, the intensity of the reflected probe, shown in Fig. 2, after the thermal excitation of the pump beam can be used to deduce the temperature profile of the system. The data is then compared to a thermal model, to be discussed in the following sections, and unknown variables are left as free parameters and varied until the sum of the least-squares differences between the data and model have been minimized.

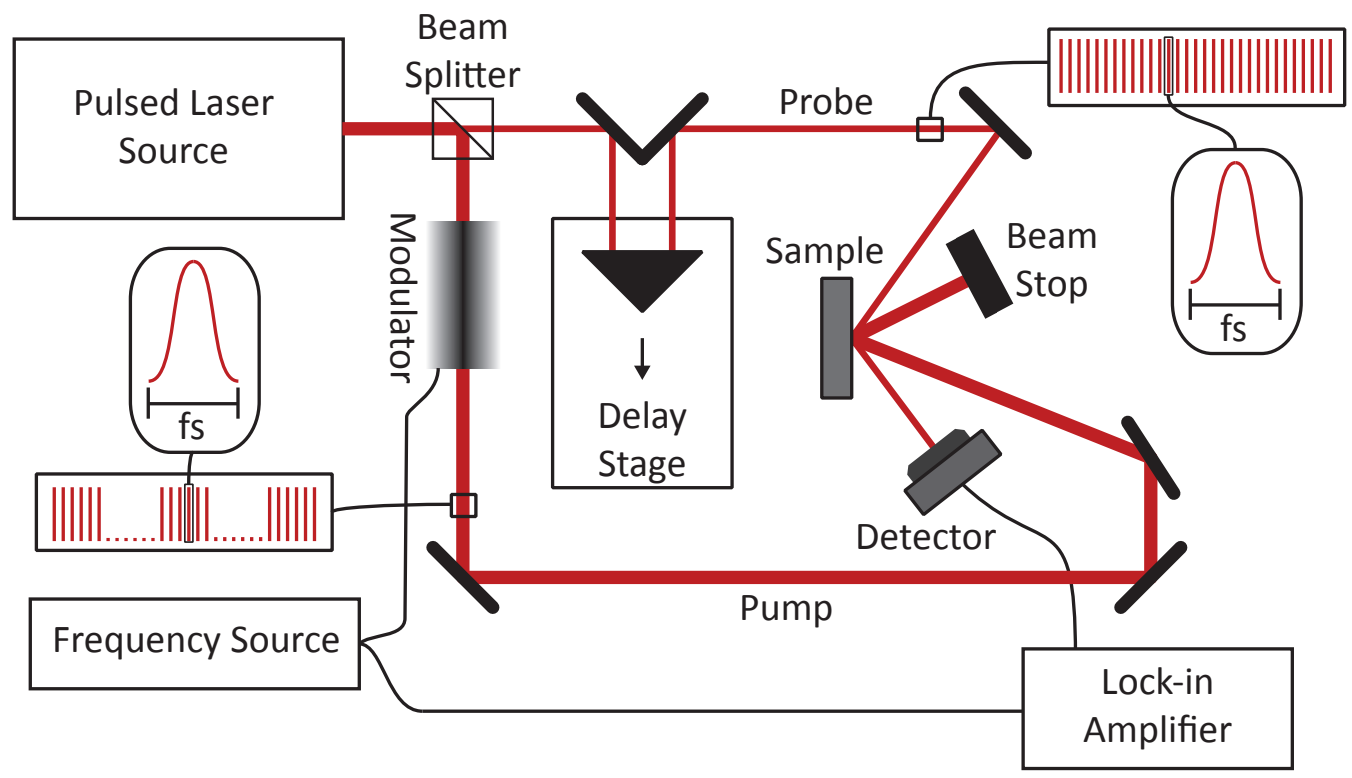

Figure 1: The basic schematic of a TDTR experimental set-up. 


\subsection{Low-Repetition Rate}

The low-repetition rate set-up used in this study is centered around a high-repetition rate $(76 \mathrm{MHz})$ Coherent MIRA 900 Ti:sapphire oscillator, the output of which is seeded into a Coherent RegA 9000 Ti:sapphire regenerative amplifier. After amplification, the emerging pulses have increased in energy from $9 \mathrm{~nJ} /$ pulse to around $4 \mu \mathrm{J} /$ pulse, at a wavelength of $800 \mathrm{~nm}$, a pulse duration of less than $200 \mathrm{fs}$, and at a repetition rate of $250 \mathrm{kHz}$. The pump beam is further modulated to $125 \mathrm{kHz}$ by a Conoptics EOM. In this system, the pump and the probe are spatially separated from one another in order to remove backscattered pump from the measured signal.

In order to infer the material's thermal properties, the temperature (reflectivity) on the sample surface must be related to a temperature gradient throughout the material. The thermal model used to analyze the data differs depending on the temporal regime of the TDTR scan. In the non-equilibrium regimes, thermalized electrons begin to cool by exchanging energy with the cold lattice system via electron-phonon scattering. The cooling of the electrons can be described by the two-temperature model (TTM), which couples the electron and lattice temperature [14]. Under the TTM, the interaction between electrons and phonons is diffusive, but ballistically traveling electrons can influence the resulting temperatures [21]. However, as we are interested in steady-state thermal properties, such as $\kappa$ and $h_{B D}$, we will focus on the transient regime of the TDTR scan, i.e., times greater than $\sim 0.5$ ns. At this time scale, the phonons and electrons (to a lesser degree in semiconductors) dominate the thermal transport through diffusive scattering processes $[22,12]$. Therefore, the heat diffusion equation can be used to relate the measured surface temperature to the thin film's thermal properties [23]. The heat diffusion equation is solved through a Crank-Nicolson $(\mathrm{CN})$ method. $\mathrm{CN}$ is a finite difference method which is numerically stable only when the value $\alpha\left(\delta t / \delta x^{2}\right)$ is large, where $\alpha$ is the thermal diffusivity, $\delta t$ is the discrete time step, and $\delta x$ is the discrete spatial step [24, 25]. Thus, small spatial and temporal steps must be used for an accurate analysis, and as with any finite element analysis model, the computational power required scales with the number of discrete steps needed. In order to greatly increase the speed of our analysis, a one-dimensional heat diffusion equation was utilized. At low-frequencies, a one-dimensional analysis can only be applied when the pump's diameter is significantly larger than the probe. In this experiment, we maintain a 5:1 ratio, which is sufficient to assume one-dimensional heat transfer [17]. In the following sections, the 1-D heat equation was solved 
using $\mathrm{CN}$ in the steady-state regime. The unknown thermal properties, such as the thermal conductivity of a substrate or the thermal boundary conductance, are left as free parameters. Using a non-linear least-squares regression analysis, we vary the unknown properties until the residuals are minimized.

\subsection{High-Repetition Rate}

The high-repetition system utilized in this work is primarily the same as described above, without the amplified cavity. The femtosecond pulsed laser

used is a Spectra Physics Tsunami, which operates at a repetition rate of $80 \mathrm{MHz}$, a pulse width less than $200 \mathrm{fs}$, and an energy/pulse around $30 \mathrm{~nJ}$. After emanating from the cavity, the pump beam is further modulated to $1 \mathrm{MHz}$. The primary difference between high and low-rep rate systems is a phase, or imaginary component, of the measured data. In low-rep systems, the sample returns to thermal equilibrium, following a pump pulse, before the arrival of a subsequent pump pulse. Consequently, the signal arriving at the detector is from one heating event, and therefore has a constant phase. In high-rep systems, the sample does not have adequate time to cool down completely before the next heating event is created and there is a resulting thermal accumulation on the sample surface, producing a changing phase signal. The phase signal has been used to provide additional sensitivity to thermal properties, such as radial thermal conductivity in anisotropic systems [26].

A similar analysis as described above for the low-repetition system is used in high-repetition systems, where a thermal model is compared to the data and unknown properties are varied until the difference between the model and data is minimized. The thermal accumulation on the sample surface complicates the analysis so that we cannot just discretely solve the $1 \mathrm{D}$ heating equation. Instead, we must account for the sinusoidal nature of the heating event, and can use a Feldman analysis for heat flow [27]. Again, the modulation frequency must be considered when determining the relative pump and probe sizes to insure cross-plane heating. In this study, a $1 / \mathrm{e}^{2}$ diameter of $48 \mu \mathrm{m}$ and $19 \mu \mathrm{m}$ were used for the pump and probe, respectively. 


\section{Results and Discussions}

\subsection{Case Study: SiC and Oxide Layer}

Silicon carbide ( $\mathrm{SiC}$ ) is a wide-bandgap semiconductor with a large thermal conductivity and breakdown field. These properties make SiC desirable for high-power electronic devices operating in high-temperature environments [28, 29]. While $\mathrm{SiC}$ possessing a native oxide, $\mathrm{SiO}_{2}$ makes it a great candidate in MOS-devices, it also produces the potential to substantially impact the resistance of the interface, which could lead to a build-up of energy carriers at an interface [30]. The presence of an oxide at the $\mathrm{Al} / \mathrm{Si}$ interface has been shown to reduce the thermal boundary conductance by a factor of six at room temperature $[31,32]$. Here, we report on the thermal boundary conductance, $\mathrm{h}_{B D}$, at room temperature $(\mathrm{T}=300 \mathrm{~K})$ at an $\mathrm{Al} / \mathrm{SiC}$ interface and an $\mathrm{Al} / \mathrm{SiO}_{2} / \mathrm{SiC}$ interface.

$\mathrm{N}$-doped $4 \mathrm{H}-\mathrm{SiC}$ was grown epitaxially a top a substrate of $\mathrm{SiC}$. The films of $\mathrm{SiC}$ are $11 \mu \mathrm{m}$ thick with doping concentrations of $10^{15} \mathrm{~cm}^{-3}$. The $\mathrm{SiC}$ was thermally oxidized, and then annealed in an ambient $\mathrm{NO}$ environment at $1175^{\circ} \mathrm{C}$. Annealing in an NO process has been shown to remove excess carbon at the $\mathrm{SiC} / \mathrm{SiO}_{2}$ interface, which produces a large density of interface states that can result in charge trapping and Coulomb scattering [33, 34]. The surface of the $\mathrm{SiC}$ substrates were then etched with buffered oxide etch (BOE) 7:1. The sample was then cleaved into several pieces. Part of the sample was immediately put in an electron evaporation chamber to deposit a nominal thickness of $100 \mathrm{~nm}$ of polycrystalline Al. On the other sample, a nominal thickness of $40 \mathrm{~nm}$ of $\mathrm{SiO}_{2}$ was grown through plasma-enhanced chemical vapor deposition (PECVD). $\mathrm{N}_{2} \mathrm{O}$ and $2 \%$ silane $\left(\mathrm{SiH}_{4}\right)$ were maintained at a 2:1 flow rate, the pressure of the chamber was kept at $250 \mathrm{mTorr}$, and the growth temperature was $270^{\circ} \mathrm{C}$. With these growth parameters, the growth rate was $\sim 30 \mathrm{~nm} / \mathrm{min}$. The thickness of the PECVD $\mathrm{SiO}_{2}$ film was measured with ellipsometry and determined to be $40 \pm 1 \mathrm{~nm}$, and the index of refraction was measured to be around 1.43-1.45, just below the bulk $\mathrm{SiO}_{2}$, indicating a slightly less dense film. The films were then metallized with $100 \mathrm{~nm}$ of $\mathrm{Al}$ grown via electron beam evaporation. The samples were then thermally characterized with both the high-rep and low-rep laser systems. Six scans were collected on each of the samples at room temperature and the values of thermal boundary conductance and thermal conductivity represent the average of those six individual fit values. The error bars on the data points 
represent the standard deviation of those six values, plus the effect of an additional $2 \mathrm{~nm}$ uncertainty in the thickness of the Al transducer.

Picosecond acoustics were collected during TDTR analysis, and used to determine the local thickness of the $\mathrm{Al}$ films $[35,36]$. In the thermal model, bulk specific heat values for the $\mathrm{Al}$ and $\mathrm{SiC}$ were assumed and a reduced thermal conductivity was assumed for $\mathrm{Al}$ (due to finite size and boundary scattering), and the values used can be found in Refs. [37, 38, 39]. We also left the thermal conductivity, $\kappa_{S i C}$ as a free parameter for the 2 layer system, $\mathrm{Al}$ /etched $\mathrm{SiC}$, and it was determined to be $323 \pm 2.0 \mathrm{~W} / \mathrm{mK}$ in the low-rep system, and $322 \pm 9.6 \mathrm{~W} / \mathrm{mK}$ using the high-rep system. These results are within $10 \%$ of the measured value by Ref. [37], which is reasonable for a doped sample. The deviation between individual measurements was much larger for the high-rep system, and we believe this is due to differences in the experimental sensitivity of the two systems. The experimental sensitivity, $S$, of a particular parameter, $\alpha$, is defined as,

$$
S_{\alpha}=\frac{d(\ln (V)}{d(\ln (\alpha))}
$$

where $V$ is the signal from TDTR [40]. In the low-rep system, we are very sensitive to both thermal conductivity of the substrate, $\kappa_{S i C}$, and the thermal boundary conductance, $h_{B D, A l / S i C}$, but in the high-rep system, the sensitivity to $\kappa_{S i C}$ is overshadowed by the sensitivity to $h_{B D, A l / S i C}$, see the inset of Fig. 3. This is likely due to a much larger thermal penetration depth in the low-rep system, since modulation frequency is inversely proportional to thermal penetration depth [18]. In Fig. 3, $\mathrm{h}_{B D, A l / S i c}$ is shown for both laser systems, the high-rep and the low-rep.

In the three layer system, $\mathrm{Al} / \mathrm{PECVD} \mathrm{SiO}_{2} / \mathrm{SiC}$, the thermal conductivity and specific heat of the PECVD film must also be determined since the transport properties of $\mathrm{SiO}_{2}$ have been shown to be sensitivities to processing parameters [41]. Again, the differing experimental sensitivity of the two systems is of importance here. In the high-rep system, the experimental sensitivity is dominated by the $\mathrm{SiO}_{2}$ layer, and we are not sensitive to the $\mathrm{SiC}$ substrate, but in the low-rep system, we are sensitive to all three layers due to an enhanced penetration depth. Therefore, in the high-rep system, we fit the specific heat of $\mathrm{SiO}_{2}$, the thermal conductivity of the $\mathrm{SiO}_{2}$, and the thermal boundary conductance of $\mathrm{Al} / \mathrm{SiO}_{2}$. In the low-repetition system, we treat the interfaces, as well as the $\mathrm{SiO}_{2}$ layer as a series of resistors in order to lump all the unknowns into a single unknown. We also leave the 
thermal conductivity of the $\mathrm{SiC}$ layer as an open parameter in the low-rep system as a method for checking the accuracy of our fits. The thermal conductivity of the $\mathrm{SiO}_{2}, \kappa_{\mathrm{SiO}_{2}}=1.26 \pm 0.1 \mathrm{~W} / \mathrm{mK}$ and the specific heat of the $\mathrm{SiO}_{2}, C=2.05 \times 10^{6} \mathrm{Jm}^{-3} \mathrm{~K}^{-1}$, as determined from the high-repetition fits. These values are very close to other studies of thermal properties in PECVD $\mathrm{SiO}_{2}$ [41]. In the low-repetition system, the thermal conductivity of the $\mathrm{SiC}$ substrate was determined to be $\kappa_{S i C}=324 \pm 5 \mathrm{~W} / \mathrm{mK}$. For the three-layer system in low-rep analysis, we are significantly more sensitive to the net resistance of the $\mathrm{Al} / \mathrm{SiO}_{2}$ relative to the $\mathrm{SiC}$ system, so the error bars on this measurement are larger than the two layer, low-rep, measurement of $\kappa_{S i C}$, but the value is within the error bars of our 2-layer measurement, and therefore, the measurement of the net resistance is justified. In Fig. 3, the net conductances of the low-rep and high-rep system are shown. In the three layer system, the total conductance is given by Eqn. 2 .

$$
h_{\text {total }}^{-1}=h_{B D, A l / S_{i O}}^{-1}+h_{\mathrm{SiO}_{2}}^{-1}
$$

The conductance of the $\mathrm{SiO}_{2}$ layer is given by $\kappa_{\mathrm{SiO}_{2}} / \mathrm{d}_{\mathrm{SiO}_{2}}$. The two fit values are almost identical, but the error bars for the high-rep system are larger due to more parameters being left as free parameters. These results demonstrate that the two major classification of TDTR systems, a single shot (low-rep) and a high-rep modulated response yield the same thermophysical properties, and only impact the sensitivity of the results. If the user is careful to ensure the beam parameters have been set-up properly and the correct analysis is being used, the thermophysical properties should not vary between a low-rep and high-rep system. As long as there is sufficient sensitivity, compared to the noise of the system, there is no reason to anticipate the thermal properties being dependent on the repetition rate of the system. As seen in Fig. 3, the presence of the PECVD oxide lowers the conductance by almost exactly 1 order of magnitude. This reduction agrees with literature that the presence of an oxide layer, particularly one with minimal intermixing at the interface, greatly reduces the interfacial conductance $[31,42,43]$.

\section{Conclusion}

In this note, we establish the robustness of transient reflectance measurements using two femtosecond pulsed laser systems with different repetition rates, one high in the $\mathrm{MHz}$ range and one low, in the $\mathrm{kHz}$ range. Both 
systems were used to measure the thermal boundary conductance of a resistive metal/oxide and a metal/semiconductor, the thermal conductivity of a highly conductive substrate, $\mathrm{SiC}$, and the thermal conductivity of a resistive oxide, $\mathrm{SiO}_{2}$.

The same properties were measured on both systems, but with different error bars associated with the measurements. We attribute the deviation in error bars to the differences in signal filtering. These results establish the versatility and universality of TDTR measurements.

\section{Acknowledgment}

The authors would like to thank J.L. Hostletler for the $\mathrm{SiC}$ samples. The authors would also like to acknowledge B.M. Foley and P.E. Hopkins for providing us with a high-repetition laser system to use. L.S. Larkin is appreciative of funding through the National Science Foundation's Graduate Research Fellowship Program. Both authors would like to acknowledge the financial support of the Air Force Office of Scientific Research under Grant. No. FA9550-14-1-0395

[1] A. Rosencwaig and A. Gersho, "Theory of photoacoustic effect with solids," Journal of Applied Physics, vol. 47, no. 1, 1976.

[2] J. Opsal, A. Rosencwaig, and D. Willenborg, "Thermal-wave detection and thin-film thickness measurements with laser beam deflection," $A p$ plied Optics, vol. 22, no. 20, pp. 3169-3176, 1983.

[3] G. Eesley, "Observation of nonequilibrium electron heating in copper," Physical Review Letters, vol. 51, no. 23, 1983.

[4] G. Eesley, "Generation of nonequilibrium electron and lattice temperatures in copper by picosecond laser pulses," Physical Review B, vol. 33, pp. 2144-2151, 1986.

[5] A. Tam and B. Sullivan, "Remote sensing applications of pulses photothermal radiometry," Applied Physics Letters, vol. 43, no. 4, 1983.

[6] C. Thomsen, J. Strait, Z. Vardeny, H. Maris, and J. Tauc, "Coherent phonon generation and detection by picosecond light pulses," Physical Review Letters, vol. 53, no. 10, 1984. 
[7] O. Wright and K. Kawashima, "Coherent phonon detection from ultrafast surface vibrations," Physical Review Letters, vol. 69, no. 11, 1992.

[8] C. Paddock and G. Eesley, "Transient thermoreflectance from thin metal films," Journal of Applied Physics, vol. 60, pp. 285-290, 1986.

[9] W. Leung and A. Tam, "Thermal diffusivity in thin films measured by noncontact single-ended pulsed-laser-induced thermal radiometry," Optics Letters, vol. 9, no. 3, 1984.

[10] D. Cahill, W. Ford, K. Goodson, G. Mahan, A. Majumdar, H. Maris, R. Merlin, and S. Phillpot, "Nanoscale thermal transport," Applied Physics Reviews, vol. 93, p. 793, 2003.

[11] D. Cahill, P. Braun, G. Chen, D. Clarke, S. Fan, K. Goodson, P. Keblinski, W. King, G. Mahan, A. Majumdar, H. Maris, S. Phillpot, E. Pop, and L. Shi, "Nanoscale thermal transport. ii. 2003-2012," Applied Physics Reviews, vol. 1, p. 011305, 2014.

[12] A. Schmidt, "Chapter 6, pump-probe thermoreflectance," Annual Review of Heat Transfer, pp. 159-181, 2013.

[13] P. Hopkins, J. Duda, B. Kaehr, X. Zhou, C.-Y. P. Yang, and R. Jones, "Ultrafast and steady-state laser heating effects on electron relaxation and phonon coupling mechanisms in thin gold films," Applied Physics Letters, vol. 103, p. 211910, 2013.

[14] J. Hohlfeld, S.-S. Wellershoff, J. Güdde, U. Conrad, V. Jähnke, and E. Matthias, "Electron and lattice dynamics following optical excitation of metals," Chemical Physics, vol. 251, pp. 237-358, 2000.

[15] S. Brorson, A. Kazeroonian, J. moodera, D. Face, T. Cheng, E. Ippen, M. Dresselhaus, and G. Dresselhaus, "Femtosecond room-temperature measurement of the electron-phonon coupling constant $\lambda$ in metallic superconductors," Physical Review Letters, vol. 64, pp. 2172-2175, 1990.

[16] L. Wang, R. Cheaito, J. Braun, A. Giri, and P. Hopkins, "Thermal conductivity measurements of non-metals via combined time- and frequency-domain thermoreflectance without a metal film transducer," Review of Scientific Instruments, vol. 87, p. 094902, 2016. 
[17] P. Hopkins, J. Serrano, L. Phinney, S. Kearney, T. Grasser, and C. Harris, "Criteria for cross-plane dominated thermal transport in multilayer thin film systems during modulated laser heating," Journal of Heat Transfer, vol. 132, p. 081302, 2010.

[18] Y. Koh and D. Cahill, "Frequency dependence of the thermal conductivity of semiconductor alloys," Physical Review B, vol. 76, p. 075207, 2007.

[19] R. Wilson and D. Cahill, "Anisotropic failure of Fourier theory in time-domain thermoreflectance experiments," Nature Communications, vol. 5, p. 5075, 2014.

[20] A. Schmidt, R. Cheaito, and M. Chiesa, "A frequency-domain thermoreflectance method for the characterization of thermal properties," Review of scientific instruments, vol. 80, p. 094901, 2009.

[21] P. Hopkins and P. Norris, "Contribution of ballistic electron transport to energy transfer during electron-phonon nonequilibrium in thin metal films," Journal of Heat Transfer, vol. 131, p. 043208, 2009.

[22] D. Cahill, K. Goodson, and A. Majumdar, "Thermometry and thermal transport in micro/nanoscale solid-state devices and structures," Journal of Heat Transfer, vol. 124, pp. 223-241, 2002.

[23] M. N. Ozisik, Heat Conduction. John Wiley and Sons Inc., 2 ed., 1993.

[24] P. Hopkins, P. Norris, R. Stevens, T. Beechem, and S. Graham, "Influence of interfacial mixing on thermal boundary conductance across a chromium/silicon interface," Journal of Heat Transfer, vol. 130, p. 062402, 2008.

[25] P. Norris, A. Caffrey, R. Stevens, J. Klopf, J. M. Jr., and A. Smith, "Femtosecond pump-probe nondestructive examination of materials," Review of Scientific Instruments, vol. 74, pp. 400-406, 2003.

[26] A. Schmidt, X. Chen, and G. Chen, "Pulse accumulation, radial heat conduction, and anisotropic thermal conductivity in pump-probe transient thermoreflectance," Review of Scientific Instruments, no. 79, p. 114902, 2008. 
[27] D. Cahill, "Analysis of heat flow in layered structures for time-domain thermoreflectance," Review of Scientific Instruments, vol. 75, no. 12, 2004 .

[28] Z. Chen, A. Ahyi, X. Zhu, M. Li, T. Isaacs-Smith, J. Williams, and L. Feldman, "MOS characteristics of C-face $4 \mathrm{H}-\mathrm{SiC}$," Journal of Electronic Materials, vol. 39, no. 5, 2010.

[29] A. Smith and J. Calame, "Impact of thin film thermophysical properties on thermal management of wide bandgap solid-state transistors," International Journal of Thermophysics, vol. 25, no. 2, 2004.

[30] E. Pop, "Energy dissipation and transport in nanoscale devices," Nano Research, vol. 3, pp. 147-169, 2010.

[31] J. Duda and P. Hopkins, "Systematically controlling Kapitza conductance via chemical etching," Applied Physics Letters, vol. 100, p. 11602, 2012 .

[32] A. Minnich, J. Johnson, A. Schmidt, K. Esfarjani, M. Dresselhaus, K. Nelson, and G. Chen, "Thermal conductivity spectroscopy technique to measure phonon mean free paths," Physical Review Letters, vol. 107, p. 095901, 2011.

[33] F. Devynck, First-principles study of defects at the $\mathrm{SiC} / \mathrm{SiO}_{2}$ interface through hybrid functionals. PhD thesis, Swiss Federal Institute of Technology in Lausanne, 2008.

[34] T. Seyller, "Electronic properties of $\mathrm{SiC}$ surfaced and interfaces: some fundamental and technological aspects," Applied Physics A, vol. 85, pp. 371-385, 2006.

[35] K. O'Hara, X. Hu, and D. Cahill, "Characterization of nanostructured metal films by picosecond acoustics and interferometry," Journal of Applied Physics, vol. 90, p. 4852, 2001.

[36] G. Hohensee, W. Hsieh, M. Losego, and D. Cahill, "Interpreting picosecond acoustics in the case of low interface stiffness," Review of Scientific Instruments, vol. 83, p. 114902, 2012. 
[37] M. Levinshtein, S. Rumyantsev, and M. Shur, eds., Properties of Advanced Semiconductor Materials: $G a N$ and $A l N$ and $I n N$ and $B N$ and $\mathrm{SiC}$ and SiGe. John Wiley and Sons Inc., 2001.

[38] E. Buyco and F. Davis, "Specific heat of aluminum from zero to its melting temperature and beyond," Journal of Chemical and Engineering Data, vol. 15, no. 4, 1970.

[39] A. Schmidt, R. Cheaito, and M. Chiesa, "Characterization of thin metal films via frequency-domain thermoreflectance," Journal of Applied Physics, vol. 107, p. 024908, 2010.

[40] B. Gundrum, D. Cahill, and R. Averback, "Thermal conductance of metal-metal interfaces," Physical Review B, vol. 72, p. 245426, 2005.

[41] Y. Ju and K. Goodson, "Process-dependent thermal transport properties of silicon-dioxide films deposited using low-pressure chemical vapor depsotion," Journal of Applied Physics, vol. 85, no. 10, pp. 7130-7134, 1999 .

[42] P. Hopkins, L. Phinney, J. Serrano, and T. Beechem, "Effects of surface roughness and oxide layer on the thermal boundary conductance at aluminum/silicon interfaces," Proceedings of ASME 2010 14th International Heat Transfer Conference, pp. 313-319, 2010.

[43] C. Gorham, K. Hattar, R. Cheaito, J. Duda, J. Gaskins, T. Beechem, J. Ihlefeld, L. Biedermann, E. Piekos, D. Medlin, and P. Hopkins, "Ion irradiation of the native oxide/silicon surface increases the thermal boundary conductance across aluminum/silicon interfaces," Physical Review B, vol. 90, p. 024301, 2014. 


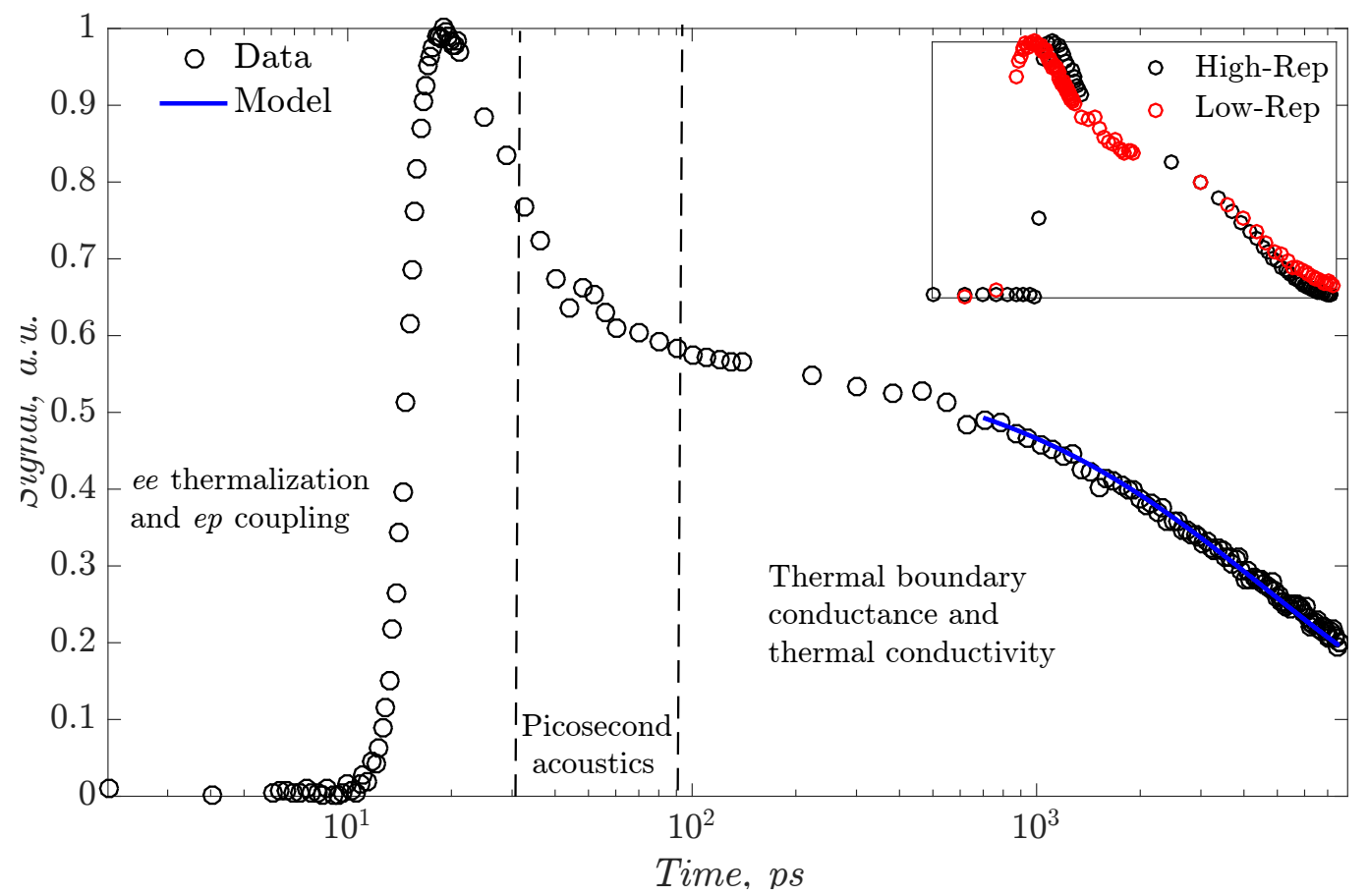

Figure 2: A sample time-domain thermoreflectance, TDTR, scan with the primary temporal regimes labeled. The first regime, the hot electron thermalization and electron-phonon coupling regime, occurs immediately after the initial excitation of the material system by the laser. Data from this regime can be used to determine a film's electron-phonon coupling constant, as well as it's optical constants. In the second regime, the picosecond acoustic regime, the data is influenced by the effects of thermal expansion in the thin metallic film following the rapid thermal excitation. These picosecond acoustic response can be used to determine the thickness within the metal film, if the speed of sound in the film is known. In the third region, heat transfer is dominated by diffusive transport, and the steady state thermal properties, such as film thermal conductivity, diffusivity, effusivity, and thermal boundary conductance can be determined. The solid blue line shows the resulting thermal model for this data. In the inset of this figure, the real component of the reflectance signal collected from the high-rep and low-rep systems are shown in black and red, respectively. 


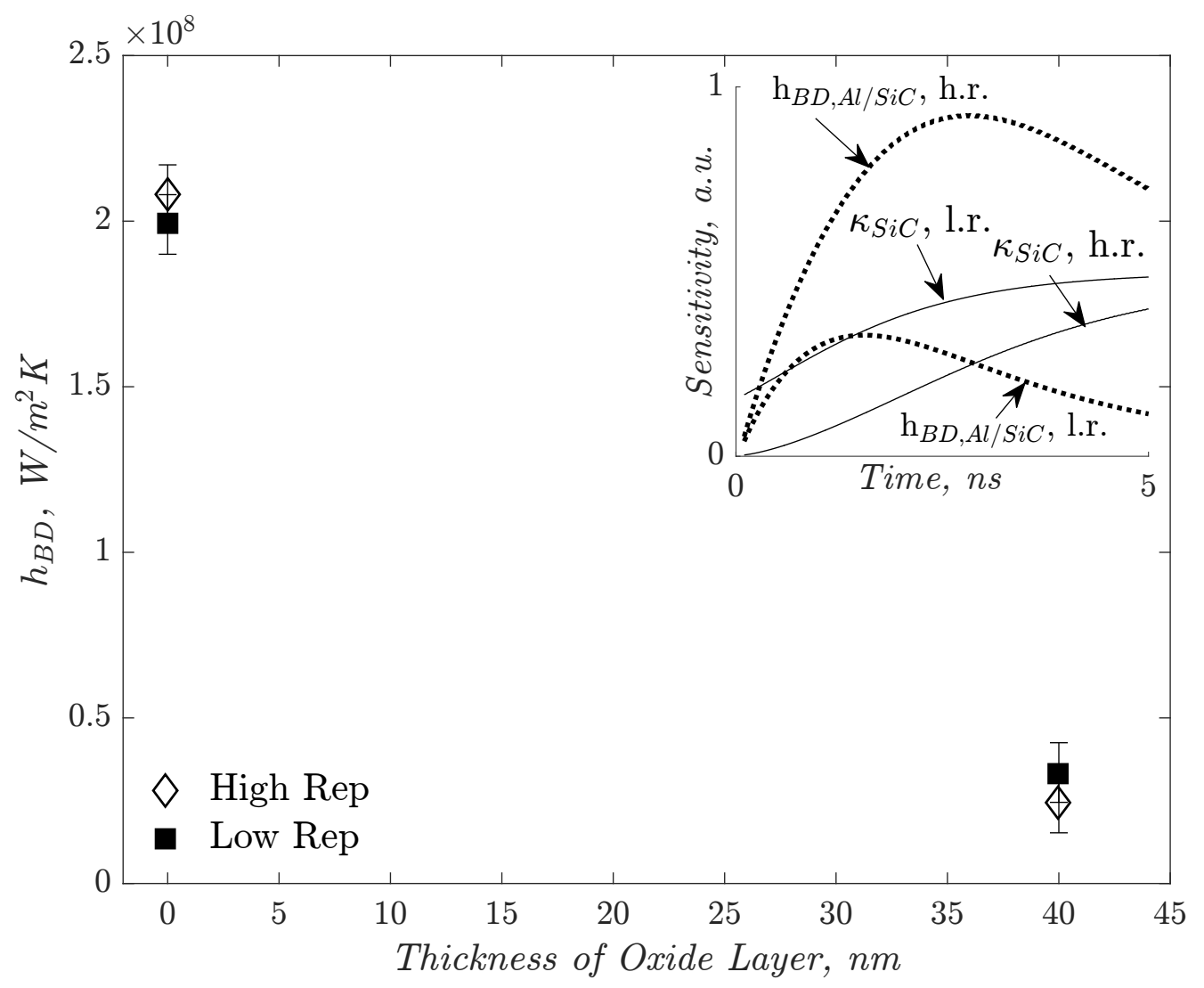

Figure 3: The thermal boundary conductance, $h_{B D, A l / S i C}$, and the total conductance, given by Eq. 2, of an $\mathrm{Al} / \mathrm{PECVD} \mathrm{SiO}_{2} / \mathrm{SiC}$ system. The measurements are shown as a function of oxide thickness, and the etched sample $(\mathrm{Al} / \mathrm{SiC})$ has a $0 \mathrm{~nm}$ oxide thickness, and the three-layer $\mathrm{Al} / \mathrm{PECVD} \mathrm{SiO}_{2} / \mathrm{SiC}$ system has a $40 \mathrm{~nm}$ oxide thickness. The measurements were performed on a both a low-repetition system, described in Sec. 2.1, marked 'Low-rep', and shown in a solid-black square, and a high-repetition system, where the set-up of this system is described in Sec 2.2, and shown here in the figure as open diamonds. The oxide layer lowers the conductance by an order of magnitude. Despite the differing experimental sensitivity of these systems, both the high-rep and low-rep system measurements are within the error bars of one another, demonstrating the robustness of TDTR. In the inset of this figure, the sensitivity for selected parameters of the 2-layer $\mathrm{Al} /$ (etched)SiC are shown for both the high-rep and low-rep system, labeled h.r. and l.r., respectively. The substantial impact of frequency on experimental sensitivity is quite pronounced. In the high-rep system, the sensitivity to $h_{B D, A l / S i C}$ greatly overshadows the sensitivity to $\kappa_{S i C}$. On the other hand, the sensitivity to $h_{B D, A l / S i C}$ and $\kappa_{S i C}$ are approximately the same for early parts of the scan, but the sensitivity to $\kappa_{S i C}$ begins to surpass at later times in the scan, when the laser has begun penetrating the $\mathrm{SiC}$ substrate. 